\title{
Impact of using artemisinin-based combination therapy (ACT) in the treatment of uncomplicated malaria from Plasmodium falciparum in a non-endemic zone
}

Joaquín Pousibet-Puerto ${ }^{1}$, Joaquín Salas-Coronas ${ }^{1,2^{*}}$, Alicia Sánchez-Crespo ${ }^{3}$, M. Angustias Molina-Arrebola ${ }^{1,3}$, Manuel J. Soriano-Pérez ${ }^{1}$, M. José Giménez-López ${ }^{3}$, José Vázquez-Villegas ${ }^{4}$ and M. Teresa Cabezas-Fernández ${ }^{1,2}$

\begin{abstract}
Background: Artemisinin-based combination therapy (ACT) has been adopted by the World Health Organization as a first-line treatment for uncomplicated Plasmodium falciparum malaria. In endemic regions, it has proven more effective in treating the disease, and even in reducing its transmission. Nonetheless, there is a scarcity of studies carried out in non-endemic areas on imported uncomplicated malaria.

Methods: This is a retrospective, observational study performed on patients diagnosed and admitted with uncomplicated P. falciparum malaria between 2004 and 2015. The objective was to compare the parasite clearance period and the average hospital length of stay for patients treated with ACT vs those receiving other treatment regimens.

Results: Eighty-five patients were included in the study. Fifty-one received ACT treatment (dihydroartemisininpiperaquine) and thirty-four patients were treated with quinine sulfate+doxycycline or atovaquone/proguanil. The parasite clearance period was shorter in the group of patients treated with ACT compared to those receiving other treatment types: $24 \mathrm{~h}$ (IQR 24) vs $48 \mathrm{~h}$ (IQR 48), p < 0.01. The average hospital stay was also shorter in the ACT group with respect to the second group: 2.67 days (IQR 1.08) vs 3.96 days (IQR 2.87), $p<0.001$. A mild case of hepatitis was registered in the group treated with ACT.
\end{abstract}

Conclusions: ACT treatment of admitted hospital patients with imported uncomplicated malaria from P. falciparum reduced the days spent hospitalized as well as producing a more rapid parasite clearance compared to classic treatment. In spite of being treated with safe medications, one has to be alert to possible adverse effects such as hepatitis and delayed haemolytic anaemia.

Keywords: Malaria, Uncomplicated malaria, Plasmodium falciparum, Artemisinin-based combination therapy, Artemisinin, Dihydroartemisinin-piperaquine

\section{Background}

Malaria is the most important parasitic disease worldwide. It has been calculated that each year is it responsible for new episodes in around 214 million patients, causing the death of approximately 438,000 the majority of which are children under 5 years of age and pregnant

\footnotetext{
*Correspondence: joaquinsalascoronas@yahoo.es

${ }^{1}$ Tropical Medicine Unit, Hospital de Poniente, El Ejido, Almería, Spain Full list of author information is available at the end of the article
}

women $[1,2]$, not to mention incalculable economic loss in endemic countries. In Europe, apart from isolated cases in Greece, malaria continues to be exclusively imported, with approximately 11,000 cases reported each year, making it the most important tropical disease on this continent [3-6]. Most imported malaria cases (as many as $88-98 \%$ ) are diagnosed in recently-arrived immigrants or resident immigrants who return to their country of origin to visit friends or family visiting friends and relatives (VFR); sub-Saharan Africa being the region 
from which most cases originate. Eighty percent of declared imported cases of malaria in Europe are $P$. falciparum [3-5].

Malaria treatment has changed substantially over recent years [7]. Artesunate has proven to be superior to quinine in most situations where complicated malaria is treated [8-12]. In uncomplicated malaria, regimens known as artemisinin-based combination therapy (ACT) demonstrates greater efficacy in treating the disease, reducing transmission in endemic areas and producing lower levels of reinfection [13-17]. For this reason, artemisinin derivatives are currently recommended as first-line medication for P. falciparum malaria treatment, whether complicated (severe) or uncomplicated, both in endemic and non-endemic countries [18-20].

Artemisinin treatment leads to a reduction in average hospital stays, more rapid parasite clearance in comparison with any other anti-malarial $[11,12,17]$; and, in complicated malaria, a lowering of the global mortality rate $[8,9]$. The majority of these studies have been carried out in endemic areas. In non-endemic countries, in patients with complicated malaria, treatment with artesunate additionally reduced the days spent in intensive care units (ICU) [11, 12]. However, in patients with uncomplicated malaria from $P$. falciparum, there are few studies comparing the differences in non-endemic regions [17].

This study aims to analyse the impact that the use of ACT has had compared to two classic treatments (quinine sulfate and doxycycline/clindamycin or atovaquoneproguanil) to treat patients admitted with uncomplicated malaria from $P$. falciparum to a hospital in a non-endemic area.

\section{Methods}

A retrospective observational study was carried out on all patients admitted with malaria to Hospital Poniente (El Ejido, Almería. Spain) from January 2004 to December 2015. The hospital serves a population of approximately 250,000 people, in an area with a large African immigrant population, many of whom work in horticultural greenhouses. The study included patients over 14 years of age whose reason for hospital admission was exclusively the diagnosis of uncomplicated malaria from P. falciparum [19]. Pregnant women and HIV patients were excluded from the study. The disease was diagnosed using direct thin and thick smear microscopy in tandem with a rapid diagnostic test (SD Bioline malaria $\mathrm{Ag} \mathrm{Pf} / \mathrm{Pan}{ }^{\circledR}$ ) using lateral flow assays. The malarial PCR (semi-nested multiplex PCR) was used only in cases where a more precise confirmation was needed of the species, or where there was a suspicion of mixed parasitic infection. A clinical history and complete physical examination were carried out on all patients, gathering epidemiological and clinical data. On admission, screening was performed for the most prevalent imported pathologies; this included HIV, $\mathrm{HBV}$ and HCV blood tests. Parasitaemia monitoring was carried out via a daily smear until a negative result was obtained. After leaving hospital, the patients were checked with a blood test and a blood smear at 7 and 28 days.

Uncomplicated malaria is defined following the recommendations for the management of imported malaria in Europe [19]. With regard to parasitaemia, patients who came from endemic countries (semi-immune patients) were considered uncomplicated if they had a parasitaemia level of $<5$ and $<2 \%$ in non-immune individuals. Dihydroartemisinin-piperaquine (Eurartesim ${ }^{\circledR}$ $320 \mathrm{mg} / 40 \mathrm{mg}$, Sigma Tau) was the ACT chosen; this medication is currently the commercial form available in Spain.

Variables were analysed corresponding to epidemiological, clinical and analytical characteristics as well as the parasite clearance period and the average hospital stay. The level of parasitaemia was divided into patients with $<1 \%$, between 1 and $2 \%$, and $>2-5 \%$. Any possible adverse secondary effects associated with the treatment were also recorded.

Data were analysed using the statistical software package SPSS v17. A descriptive analysis was carried out on the quantitative variables using the mean and the standard deviation, as well as the range. Qualitative variables were described using absolute frequencies and percentages. To observe the possible differences between the group of patients treated with quinine and doxycycline/ clindamycin or atovaquone-proguanil and ACT, the Pearson's Chi square test or Fisher's exact test was used when the variables were qualitative. When the variables for comparison were quantitative, the mean differences were estimated using the Student's t test for independent samples if they complied with the normal hypothesis and the Mann-Whitney U test when they did not.

A multivariate linear regression model was performed using days of hospitalization as the dependent variable and the treatment group, the haemoglobin $(\mathrm{Hb})$ and platelet levels (at the time of diagnosis) and the level of parasitaemia as the independent variables. The goodness-of-fit was calculated along with the determination coefficient. For all tests, the fixed level of significance was 0.05 . This study was approved by the local ethic committee (Almería, Spain) with code 12/2016.

\section{Results}

A total of 85 patients diagnosed with uncomplicated malaria were included in the study. The pharmaceuticals used for malaria treatment were quinine sulfate+doxycycline or atovaquone/proguanil until 
2012. In the same year, dihydroartemisinin-piperaquine was introduced, remaining as the first-line treatment since then. Fifty-one patients were treated with dihydroartemisinin-piperaquine and 34 with quinine sulfate+doxycycline $(\mathrm{n}=33)$ or atovaquone/proguanil $(\mathrm{n}=1)$. The dataset supporting the conclusions of this study is included as Additional file 1.

The general patient characteristics, both together and by groups, are shown in Table 1 . The average age was 32 years (IQR 9), $96.5 \%$ of which were male $(n=82)$. All of the patients except one (a Spanish expatriate) originated from sub-Saharan Africa, and 94.1\% were treated as VFR patients. No differences were observed on admittance between the study groups with relation to age, gender, country of origin, type of traveler, or basal $\mathrm{Hb}$ and platelet levels, nor the level of parasitaemia. Regarding concomitant diseases, one patient was diabetic (in the quinine sulfate+doxycycline group), two presented with arterial hypertension (in the quinine sulfate+doxycycline group), and ten patients presented with chronic hepatitis B (five in the quinine sulfate+doxycycline group and five in the dihydroartemisinin-piperaquine group).

The global parasite clearance time was $24 \mathrm{~h}$ (IQR 24) and the average hospital stay was 2.88 days (IQR 1.88). In the subgroup analysis, the parasite clearance time was significantly less in the patient group treated with ACT rather than the classic treatment: $24 \mathrm{~h}$ (IQR 24) vs $48 \mathrm{~h}$ (IQR 48), $\mathrm{p}<0.01$. The average stay was also less in the artemisinin group with respect to the group treated with quinine+doxycycline/atovaquone-proguanil: 2.67 days (IQR 1.08) vs 3.96 days (IQR 2.87), p < 0.001 (Table 1; Fig. 1).

With regard to the $\mathrm{Hb}$ levels during the follow-up at 7 and 28 days, patients treated with dihydroartemisininpiperaquine presented higher levels on both occasions (ACT group vs quinine+doxycycline/atovaquone-proguanil: day 7: $13.37 \mathrm{~g} / \mathrm{dL} \pm 1.48$ (range $10-16$ ) vs $12.65 \mathrm{~g} /$ $\mathrm{dL} \pm 1.42$ (range 10-15.5), $\mathrm{p}=0.03$; day 28: $14.32 \mathrm{~g} /$ $\mathrm{dL} \pm 1.2$ (range $11.7-16.8$ ) vs $13.45 \mathrm{~g} / \mathrm{dL} \pm 1.38$ (range 9.1-14.8), $\mathrm{p}=0.02$.

In the lineal regression model (Table 2), the variables showing an influence on the average days of

Table 1 Characteristics of the patients included in the study

\begin{tabular}{|c|c|c|c|c|}
\hline & All $N=85$ & $\begin{array}{l}\text { Quinine/atovaquone- } \\
\text { proguanil } n=34\end{array}$ & $\begin{array}{l}\text { Dihydroartemisinin- } \\
\text { piperaquine } n=51\end{array}$ & p value \\
\hline \multicolumn{5}{|l|}{ Age (years); median } \\
\hline$(\mathrm{IQR})$ & $32(9)$ & $31.5(8)$ & $33(10)$ & 0.99 \\
\hline \multicolumn{5}{|l|}{ Gender } \\
\hline Male & $82(96.5 \%)$ & $32(94.1 \%)$ & $50(98 \%)$ & 0.56 \\
\hline Female & $3(3.5 \%)$ & $2(5.9 \%)$ & $1(2 \%)$ & \\
\hline \multicolumn{5}{|l|}{ Origin } \\
\hline Sub-Saharan Africa & $84(98.8 \%)$ & $34(100 \%)$ & $50(98 \%)$ & 0.49 \\
\hline Spain & $1(1.2 \%)$ & 0 & $1(2 \%)$ & \\
\hline \multicolumn{5}{|l|}{ Type of traveller } \\
\hline VFR & $80(94.1 \%)$ & $31(91.2 \%)$ & $49(96 \%)$ & 0.25 \\
\hline Recent arrival & $4(4.7 \%)$ & $3(8.8 \%)$ & $1(2 \%)$ & \\
\hline Expatriate & $1(1.2 \%)$ & 0 & $1(2 \%)$ & \\
\hline $\begin{array}{l}\text { Hb on admission }(\mathrm{g} / \mathrm{dL}) \pm \text { typical deviation } \\
\quad \text { (range) }\end{array}$ & $13.7 \pm 1.5(10.1-16.9)$ & $13.5 \pm 1.5(10.1-16.9)$ & $13.8 \pm 1.4(10.1-16.4)$ & 0.36 \\
\hline Hb day 7 (g/dL) \pm typical deviation (range) & $13.08 \pm 1.49(10-16)$ & $12.65 \pm 1.42(10-15.5)$ & $13.37 \pm 1.48(10-16)$ & 0.03 \\
\hline Hb day 28 (g/dL) \pm typical deviation (range) & $13.94 \pm 1.34(9.1-16.8)$ & $13.45 \pm 1.38(9.1-14.8)$ & $14.32 \pm 1.2(11.7-16.8)$ & 0.02 \\
\hline Total platelets $\times 10^{3} / \mu \mathrm{L} ;$ median (IQR) & $94(60)$ & $93(59)$ & $95(81)$ & 0.93 \\
\hline \multicolumn{5}{|l|}{ Parasitaemia level (\%) } \\
\hline$<1$ & $48(56.5 \%)$ & $18(52.9 \%)$ & $30(58.8 \%)$ & 0.22 \\
\hline $1-2$ & $26(30.6 \%)$ & $9(26.5 \%)$ & $17(33.3 \%)$ & \\
\hline$>2-5$ & $11(12.9 \%)$ & $7(20.6 \%)$ & $4(7.8 \%)$ & \\
\hline $\begin{array}{l}\text { Time in which parasitaemia becomes nega- } \\
\text { tive. Hours; median }(\mathrm{IQR})^{\mathrm{a}}\end{array}$ & $24(24)$ & $48(48)$ & $24(24)$ & $<0.01$ \\
\hline Period of hospitalization. Days; median (IQR) & $2.88(1.88)$ & $3.96(2.87)$ & $2.67(1.08)$ & $<0.001$ \\
\hline
\end{tabular}




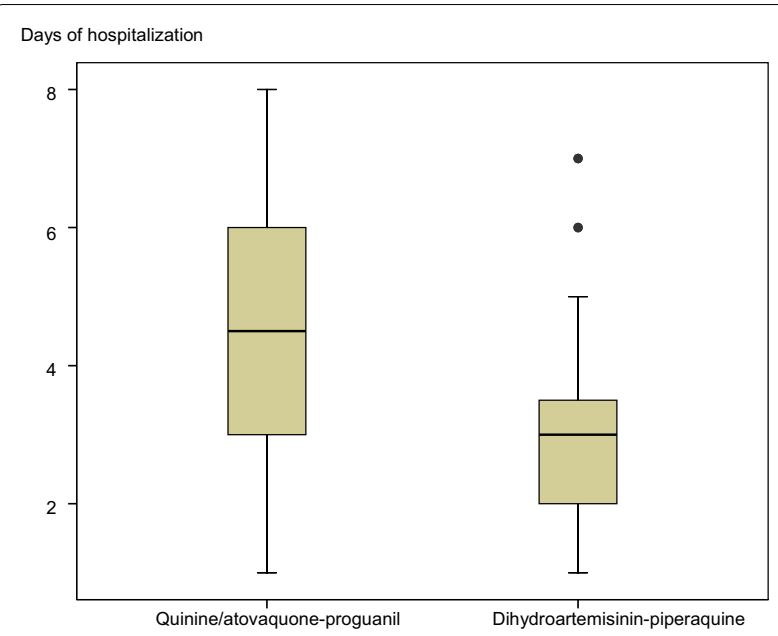

Fig. 1 Days of hospitalization per treatment group

hospitalization were the $\mathrm{Hb}$ and platelet levels at the time of admission and whether there was elevated parasitaemia $(>2-5 \%)$. The variable with the greatest association power was the treatment group, with a typified coefficient of -0.409 . For the same $\mathrm{Hb}$, platelet and parasitaemia levels, the use of ACT resulted in a reduction in hospital stay of almost a day and a half ( -1.35 days).

With regard to any reported adverse effects, in the group treated with dihydroartemisinin-piperaquine, there was one mild case of hepatitis, in the only non-subSaharan patient; this was self-limiting but, nevertheless, led to more days spent in hospital (6 days).

\section{Discussion}

ACT is a very effective and safe treatment in the management of imported uncomplicated malaria. Besides producing more rapid parasite clearance, its use in patients admitted with uncomplicated malaria reduces the period of hospitalization compared to classic treatments that were used previously, resulting in healthcare savings.
The management of imported malaria is still a challenge for many healthcare professionals in non-endemic zones. $P$. falciparum is the species most frequently involved, and also the one which most results in morbidity and/ or mortality in travellers (tourists and expatriates), as well as in foreigners who return home to visit friends and family [3-5]. The use of artemisinin derivatives has been spreading, various articles have been published showing the superior results of these drugs compared to other treatments; indeed, their use has been recommended as a first-line drug in the main national and international guidelines [18-20]. Quinine is now relegated to a secondline treatment for cases where contraindications exist, or because of the unavailability of ACT or atovaquone-proguanil; likewise in complicated malaria treatment given within the first trimester of pregnancy due to the possibility of teratogenicity $[7,18-20]$.

Most studies concerning imported malaria have been carried out on patients with complicated malaria $[11,12]$ with artesunate being the most-commonly used drug for intravenous treatment. Besides producing faster parasite clearance and ending fever, it has a better safety profile (posing predominantly less risk of severe hypoglycaemia), shortens hospitalization and has recently been shown to reduce the length of time in ICU, above all in patients with more elevated parasitaemia [12]. No reduction in mortality has been demonstrated given the few patients who died in the studies thus making such analysis impossible, although with the solid evidence obtained in endemic regions, it would be unethical to carry out clinical trials with this objective in mind.

In the case of uncomplicated malaria caused by $P$. falciparum, the general recommendation is hospital admittance and oral treatment for at least 24-48 h [20], with the aim of observing the clinical evolution and tolerance to the drugs. Very few studies have been carried out with ACT in non-endemic zones. Hatz et al. [21] concluded that treatment with artemether-lumefantrine is safe and efficacious in those patients. A prospective observational

Table 2 Linear regression model

\begin{tabular}{|c|c|c|c|c|}
\hline & \multicolumn{2}{|c|}{ Coefficient } & \multicolumn{2}{|c|}{ Typified coefficient } \\
\hline & B & Typical error & Beta & Significance \\
\hline Treatment with quinine/atovaquone-proguanil or ACT & -1.349 & 0.293 & -0.409 & $<0.001$ \\
\hline $\mathrm{Hb}$ on admission & 0.314 & 0.097 & 0.284 & $<0.01$ \\
\hline Platelets on admission & -0.007 & 0.002 & -0.258 & $<0.01$ \\
\hline Average parasitaemia & -0.009 & 0.319 & -0.003 & 0.98 \\
\hline High parasitaemia & 1.083 & 0.449 & 0.225 & 0.02 \\
\hline
\end{tabular}

ACT artemisinin-based combination therapy

$R^{2}=0.404(p<0.001)$. The model we have constructed explains $40.4 \%$ of the variability in the average hospital stay. The variables that influence the average stay are the type of treatment, $\mathrm{Hb}$, platelets and having elevated parasitaemia. The variable with the greatest power of association is the treatment type, which has a typified coefficient of 0.409. For the same level of $\mathrm{Hb}$, platelets or parasitaemia, the use of ACT results in almost 1 day less of hospitalization ( -1.35 days) 
multicentre study (MALTHER) compared the various treatment regimens for imported uncomplicated malaria in Europe [17]. There were a total of 18 different regimens although the most used were atovaquone-proguanil, quinine and artemether-lumefantrine. Compared to other regimens, quinine was associated with the highest non-completion rate, more secondary effects and the most days of hospitalization. In contrast, the artemethermefloquine combination was linked to the fastest parasite clearance and cessation of fever. Regarding the period of hospitalization, the use of quinine was associated with longer hospital stays. In this study, unlike the MALTHER study, dihydroartemisinin-piperaquine was used as the $\mathrm{ACT}$, the only drug of its type commercially available in Spain and presumably the main drug used in the majority of Spanish hospitals; this combination has also been demonstrated to be efficacious and safe both in endemic countries as well as in Europe [16, 22]. In addition, as belonging to just one centre, the two groups of patients compared were much more homogenous thus avoiding, to a large extent, any bias derived from variations in clinical practice between healthcare sites or in different countries.

The overall average hospital stay for patients in our study was 2.88 days. The fact that most of the patients are immigrants has probably contributed to a slightly longer stay to that recommended. The language barrier that exists with many of these patients, as well as the problems concerning frequent follow-up visits to the healthcare centres, often for economic or work-related reasons, means that it is common their doctors feel more comfortable keeping them admitted to hospital until they are sure of good treatment tolerance and response. The rapid parasite clearance and the markedly shorter treatment regimen using $\mathrm{ACT}$ are probably the factors that most influence the length of stay.

Atovaquone-proguanil is another medication recommended as a first-line treatment for uncomplicated malaria from P. falciparum [18-20]; however, the parasite clearance rate is longer than with ACT and the adverse effects, above all gastrointestinal, are similarly more frequent [17]. Furthermore, ACT has a very good profile in relation to possible Plasmodium resistance.

With regard to possible adverse reactions, the compounds derived from artemisinin have shown good tolerance and have a good safety profile [23, 24]. The most frequent adverse effects are: Type 1 hypersensitivity at the cutaneous level; a long QTc interval in the ECG; and, at the gastrointestinal level, nausea, vomiting and diarrhoea, although often this can be clinically confused with the malaria itself [25]. Cases have also been described of raised transaminase levels or hepatitis. This was only observed in one patient; and although there were no important consequences and the condition ceased after only a few days, it did result in more days spent in hospital.

The most severe adverse effect of artemisinin derivatives however is delayed haemolytic anaemia [25-28]. This is produced by the "pitting" mechanism, in which the parasitized erythrocytes, after being cleared of parasites by the spleen, are resealed and returned to the blood stream, but with a reduced lifespan of 1-3 weeks [28]. This lysis process occurs on average 2 weeks after the drug is administered, and in some cases can require a blood transfusion. Although this has mainly been seen in patients with complicated malaria (with elevated parasitaemia) treated with artesunate, it has also been described in patients treated with intramuscular artemether and oral artemetherlumefantrine [27]. As far as it is known, no case has been reported in relation to dihydroartemisinin-piperaquine. In this study, the $\mathrm{Hb}$ levels were higher even in the group treated with ACT at 7 and 28 days. This is probably because in patients with uncomplicated malaria, delayed haemolysis is the exception; in fact, the more rapidly the parasitaemia disappears and the ACT-treated patients recover, the more rapid the accompanying recovery of the basal hemoglobin levels as well.

With regard to pregnancy, there is insufficient information about the safety and efficacy of many anti-malarials, especially in the first trimester. Consequently, the only drugs considered safe are quinine, chloroquine, clindamycin and proguanil. Artemisinin-derived drugs are safe and efficacious in the second and third trimesters, in that they have been demonstrated not to be teratogenic $[29,30]$.

The limitations present in this study come from the fact that it is a retrospective work.

Furthermore, given the characteristics of the population that is the object of the study, the results may only be strictly extrapolated to African immigrants treated with two treatment regimens, one which has already passed to second-line use (quinine sulfate-doxycycline) and the other, an ACT (dihydroartemisinin-piperaquine), which is a first-line treatment. New prospective studies will need to be carried out to evaluate if any differences exist in terms of safety, efficacy and healthcare costs between the ACT-based treatments and, for example, atovaquoneproguanil, which is also a fixed combination over the same period ( 3 days in total). Moreover, with atovaquoneproguanil, ample experience has been gathered in nonendemic regions and it has a proven safety record.

\section{Conclusions}

The results of this work reinforce the results of the few studies published to date regarding ACT treatments for patients with uncomplicated malaria in non-endemic 
regions. They are drugs with a good safety record, clearing parasitaemia more rapidly than other anti-malarials, and are probably more cost effective by reducing the average length of stay in hospital. Nonetheless, despite being safe drugs, one should be alert to the possibility of adverse effects such as hepatitis and delayed haemolytic anaemia.

\section{Additional file}

Additional file 1. General data table.

\begin{abstract}
Abbreviations
ACT: artemisinin-based combination therapy; $\mathrm{Hb}$ : haemoglobin; HBV: hepatitis $B$ virus; $\mathrm{HCV}$ : hepatitis C virus; HIV: human immunodeficiency virus; ICU: intensive care unit; IQR: interquartile rate; PCR: polymerase chain reaction; VFR: visiting friends and relatives.

\section{Authors' contributions}

JPP participated in the analytical design, interpretation, writing the draft of the manuscript and revisions. JSC contributed to the conception and design of the project, analytical design, interpretation, writing the draft of the manuscript and revisions. ASC contributed to the data collection, writing and revising the manuscript. MAMA contributed to the conception and design, writing and revising the manuscript. MJSP contributed to contributed to the data collection and revising the manuscript. MJGL contributed to the data collection, writing and revising the manuscript. JWV contributed to the data collection and revising the manuscript. MTCF contributed to the design, writing and revising the manuscript. All authors read and approved the final manuscript.
\end{abstract}

\section{Author details}

${ }^{1}$ Tropical Medicine Unit, Hospital de Poniente, El Ejido, Almería, Spain. ${ }^{2}$ Center for the Study of Migration and Intercultural Relations (CEMyRI), University of Almeria, Almería, Spain. ${ }^{3}$ Haematology Unit, Hospital de Poniente, El Ejido, Almería, Spain. ${ }^{4}$ Tropical Medicine Unit, Distrito Poniente, Almería, Spain.

\section{Acknowledgements}

We thank Pablo Garrido Fernández and Ma del Carmen Olvera Porcel, technicians in Research Methodology and Biostatistics from FIBAO, for providing invaluable support for the statistical analysis of the data.

\section{Competing interests}

JSC, MJSP and JVV participate in the pharmacoepidemiological studies "Safety Registry for Eurartesim " ${ }^{\mathrm{TM}_{\text {" }}}$ and "Pregnancy Registry for Eurartesim ${ }^{\mathrm{TM}_{\text {" }}}$. The others authors have declared that there are no competing interests.

\section{Availability of data and materials}

The dataset supporting this article's conclusions is included as Additional file 1.

\section{Ethics approval and consent to participate}

This is a retrospective study. We used the TMU (Tropical Medicine Unit) database (electronic). Data was exported to a new SSPS file. Subjects were anonymously coded, unlinked from any information identifying the source individuals. Although the study was retrospective and no action on patients was involved, the study protocol was nevertheless submitted to the Ethics Committee of de Coordinating Site (Comité de Ética de la Investigación de Almería) for approval. The latter acknowledged the study protocol and formally authorized the study (protocol no 12/2016 of 30th March, 2016).

\section{Funding}

This study has been conducted within the activities developed by the research group PAIDI CTS 582 of the regional Ministry of Gender, Health and Social Policy of the Government of Andalusia, and the CEMyRI (Centre for the Study of Migration and Intercultural Relations) of the University of Almeria
(Spain). This retrospective study has not had costs because it is a study based on clinical practice.

Received: 4 April 2016 Accepted: 20 June 2016

Published online: 02 July 2016

References

1. WHO. World malaria report. Geneva: World Health Organization; 2015.

2. Murray CJ, Rosenfeld LC, Lim SS, Andrews KG, Foreman KJ, Haring D, et al. Global malaria mortality between 1980 and 2010: a systematic analysis. Lancet. 2012:379:413-31.

3. Schlagenhauf P, Weld L, Goorhuis A, Gautret P, Weber R, von Sonnenburg F, et al. Travel-associated infection presenting in Europe (2008-12): an analysis of EuroTravNet longitudinal, surveillance data, and evaluation of the effect of the pre-travel consultation. Lancet Infect Dis. 2015;15:55-64.

4. Ramirez-Olivencia G, Herrero MD, Subirats $M$, de Juanes JR, Peña JM, S Puente. Imported malaria in adults. Clinical, epidemiological and analytical features. Rev Clin Esp. 2012;212:1-9.

5. Behrens $\mathrm{RH}$, Neave $\mathrm{PE}$, Jones CO. Imported malaria among people who travel to visit friends and relatives: is current UK policy effective or does it need a strategic change? Malar J. 2015;14:149.

6. Tseroni M, Baka A, Kapizioni C, Snounou G, Tsiodras S, Charvalakou M, et al. Prevention of malaria resurgence in greece through the association of mass drug administration (MDA) to immigrants from malaria-endemic regions and standard control measures. PLoS Negl Trop Dis. 2015;19(9):e0004215.

7. Achan J, Talisuna AO, Erhart A, Yeka A, Tibenderana JK, Baliraine FN, et al. Quinine, an old anti-malarial drug in a modern world: role in the treatment of malaria. Malar J. 2011;10:144.

8. Dondorp AM, Fanello Cl, Hendriksen IC, Gomes E, Seni A, Chhaganlal KD, et al. South East Asian quinine artesunate malaria trial (SEAQUAMAT) group. Artesunate versus quinine for treatment of severe falciparum malaria: a randomised trial. Lancet. 2005;366:717-25.

9. Dondorp AM, Fanello Cl, Hendriksen IC, Gomes E, Seni A, Chhaganlal KD, et al. Artesunate versus quinine in the treatment of severe falciparum malaria in African children (AQUAMAT): an open-label, randomised trial. Lancet. 2010;376:1647-57.

10. Maka DE, Chiabi A, Ndikum V, Achu D, Mah E, Nguefack S, et al. A randomized trial of the efficacy of artesunate and three quinine regimens in the treatment of severe malaria in children at the Ebolowa Regional Hospital. Cameroon. Malar J. 2015;14:429.

11. Eder M, Farne H, Cargill T, Abbara A, Davidson RN. Intravenous artesunate versus intravenous quinine in the treatment of severe falciparum malaria: a retrospective evaluation from a UK centre. Pathog Glob Health. 2012;106:181-7.

12. Kurth F, Develoux M, Mechain M, Clerinx J, Antinori S, Gjørup IE, et al. Intravenous artesunate reduces parasite clearance time, duration of intensive care and hospital treatment in patients with severe malaria in Europe: the tropnet severe malaria study. Clin Infect Dis. 2015;61:1441-4.

13. Sagara I, Fofana B, Gaudart J, Sidibe B, Togo A, Toure S, et al. Repeated artemisinin-based combination therapies in a malaria hyperendemic area of Mali: efficacy, safety, and public health impact. Am J Trop Med Hyg. 2012;87:50-6.

14. Abay SM. Blocking malaria transmission to Anopheles mosquitoes using artemisinin derivatives and primaquine: a systematic review and metaanalysis. Parasit Vectors. 2013;6:278.

15. Zwang J, Olliaro P, Barennes H, Bonnet M, Brasseur P, Bukirwa H, et al. Efficacy of artesunate-amodiaquine for treating uncomplicated falciparum malaria in sub-Saharan Africa: a multi-centre analysis. Malar J. 2009;8:203.

16. Adjei A, Narh-Bana S, Amu A, Kukula V, Nagai RA, Owusu-Agyei S, et al. Treatment outcomes in a safety observational study of dihydroartemisinin/piperaquine (Eurartesim $\left({ }^{\circledR}\right)$ in the treatment of uncomplicated malaria at public health facilities in four African countries. Malar J. 2016:15:43.

17. Bouchaud O, Mühlberger N, Parola P, Calleri G, Matteelli A, Peyerl-Hoffmann $G$, et al. Therapy of uncomplicated falciparum malaria in Europe: MALTHER - a prospective observational multicentre study. Malar J. 2012;11:212.

18. WHO. Guidelines for the treatment of malaria. 3rd ed. Geneva: World Health Organization; 2015. 
19. Askling HH, Bruneel F, Burchard G, Castelli F, Chiodini PL, Grobusch MP, et al. Management of imported malaria in Europe. Malar J. 2012;11:328.

20. Muñoz J, Rojo-Marcos G, Ramírez-Olivencia G, Salas-Coronas J, Treviño B, Perez Arellano IL, et al. Diagnóstico y tratamiento de la malaria importada en España: recomendaciones del Grupo de Trabajo de Malaria de la Sociedad Española de Medicina Tropical y Salud Internacional (SEMTSI). Enferm Infecc Microbiol Clin. 2015;33:e1-13.

21. Hatz C, Soto J, Nothdurft HD, Zoller T, Weitzel T, Loutan L, et al. Treatment of acute uncomplicated falciparum malaria with artemether-lumefantrine in nonimmune populations: a safety, efficacy, and pharmacokinetic study. Am J Trop Med Hyg. 2008:78:241-7.

22. Le Garlantezec P, Richard C, Broto H, Rapp C. Treatment of imported Plasmodium falciparum malaria: role of the combination of dihydroartemisinin and piperaquine. Med Sante Trop. 2015;25:136-40.

23. Keating GM. Dihydroartemisinin/piperaquine: a review of its use in the treatment of uncomplicated Plasmodium falciparum malaria. Drug. 2012;72:937-61.

24. Nosten F, White NJ. Artemisinin-based combination treatment of falciparum malaria. Am J Trop Med Hyg. 2007;77(Suppl 6):181-92.

25. Efferth T, Kaina B. Toxicity of the antimalarial artemisinin and its derivatives. Crit Rev Toxicol. 2010;40:405-21.
26. Rolling T, Wichmann D, Schmiedel S, Burchard GD, Kluge S, Cramer JP. Artesunate versus quinine in the treatment of severe imported malaria: comparative analysis of adverse events focussing on delayed haemolysis. Malar J. 2013;12:241.

27. Rehman K, Lötsch F, Kremsner PG, Ramharter M. Haemolysis associated with the treatment of malaria with artemisinin derivatives: a systematic review of current evidence. Int J Infect Dis. 2014;29:268-73.

28. Jauréguiberry S, Ndour PA, Roussel C, Ader F, Safeukui I, Nguyen M, et al. Post-artesunate delayed hemolysis is a predictable event related to the lifesaving effect of artemisinins. Blood. 2014;124:167-75.

29. Manyando C, Kayentao K, d'Alessandro U, Okafor HU, Juma E, Hamed K. A systematic review of the safety and efficacy of artemether-lumefantrine against uncomplicated Plasmodium falciparum malaria during pregnancy. Malar J. 2012;11:141

30. Burger RJ, van Eijk AM, Bussink M, Hill J, Ter Kuile FO. Artemisinin-based combination therapy versus quinine or other combinations for treatment of uncomplicated Plasmodium falciparum malaria in the second and third trimester of pregnancy: a systematic review and meta-analysis. Open Forum Infect Dis. 2015;3:170.

\section{Submit your next manuscript to BioMed Central and we will help you at every step:}

- We accept pre-submission inquiries

- Our selector tool helps you to find the most relevant journal

- We provide round the clock customer support

- Convenient online submission

- Thorough peer review

- Inclusion in PubMed and all major indexing services

- Maximum visibility for your research

Submit your manuscript at www.biomedcentral com/submit 